\title{
ON THE NUMERICAL EVALUATION OF STOP-LOSS PREMIUMS
}

\author{
F. Covens, M. Van Wouwe and M. Goovaerts
}

\begin{abstract}
A numerical procedure is described to evaluate the stop-loss premium in case the risk process is a compound Poisson process. The method is mainly based on an algorithm of $\mathrm{R}$. Piessens and $\mathrm{M}$. Branders for the numerical evaluation of Fourier transforms.
\end{abstract}

\section{INTRODUCTION}

Until now a lot of attempts have been made for computing the stop-loss premium in the case of a compound Poisson process. Some of these procedures are exposed in a contribution of Bohman and Esscher (1963). A more recent procedure was proposed by HALMSTAD (1976). He considers a discrete claim amount distribution. His method is based on the use of generating functions. The algorithm performs good results if one is able of determining the largest claimsize, which is included in the computation of the approximated generating function.

The numerical procedure, here presented, computes the stop-loss premium for the compound Poisson process, involving a continous density function $g(x)$ for the individual claim amounts. In this sense, it can be considered as an extension of the procedure proposed by David Halmstad in the discrete risk theory. The plan of the present paper is as follows: in section 2 the analytical steps in transforming the representation of the stop-loss premium are given and applied to three different cases. The next section gives a brief discussion of the numerical procedure. The last section contains some numerical results.

\section{ANALYTICAL STEPS}

Recently SEAL (1977) described a numerical procedure to invert numerically some characteristic functions. In fact he uses a classical trapezoidal quadrature formula in connection with the also classical cosinus transform, applied on $e^{-c x} f(x)$. In fact the following set of inversion formulae hold

$$
\begin{aligned}
& \phi(u)=\int_{0}^{\infty} \cos (u x) f(x) d x \\
& f(x)=\frac{2}{\pi} \int_{0}^{\infty} \cos (u x) \phi(u) d u
\end{aligned}
$$

and, some elementary calculus shows that in case:

$$
f(x)=\int_{x}^{\infty}(v-x) d F(v, t)
$$


where $F(v, t)$ denotes the distribution function of the compound Poisson process, with cosinus transform $\varphi_{F}(u), f(x)$ can be cast into the form:

$$
f(x)=\frac{2}{\pi} \int_{0}^{\infty} \cos (u x) \frac{1-\varphi_{F}(u)}{u^{2}} d u \quad x \geqslant 0
$$

where

$$
\varphi_{F}(u)=e^{-\lambda t+\lambda t} \int_{0}^{\infty} \cos (u x) d F_{x}(x) \cos \left(\lambda t \int_{0}^{\infty} \sin (u x) d F_{x}(x)\right) .
$$

Let us consider next three special cases, namely a sum of exponentials, a gamma and a Bessel density. Successively the following results can be obtained:

(6) i)

$$
\begin{aligned}
& f_{X}(x)=\sum_{1}^{n} a_{v} \alpha_{v} e^{-\alpha_{v} x} \\
& \int_{0}^{\infty} \cos (u x) f_{X}(x) d x=\sum_{\nu=1}^{n} a_{\nu} \alpha_{v} \frac{\alpha_{\nu}}{\alpha_{v}^{2}+u^{2}} \\
& \int_{0}^{\infty} \sin (u x) f_{X}(x) d x=\sum_{v=1}^{n} a_{v} \alpha_{\nu}-\frac{u}{\alpha_{\nu}^{2}+u^{2}} .
\end{aligned}
$$

These results can be inserted in the r.h.s. of (5) in order to get an elegant analytical expression for the Fourier transform $\varphi_{F}(u)$.

$$
f_{X}(x)=e^{-x_{X^{\nu}}-1} / \Gamma(\nu) .
$$

Again performing an elementary calculation gives:

$$
\begin{aligned}
& \int_{0}^{\infty} \cos (u x) f_{X}(x) d x=\frac{1}{\left(1+u^{2}\right)^{v / 2}} \cos (\nu \operatorname{Arctg} u) \\
& \int_{0}^{\infty} \sin (u x) f_{X}(x) d x=\frac{1}{\left(1+u^{2}\right)^{\nu / 2}} \sin (\nu \operatorname{Arctg} u)
\end{aligned}
$$

consequently the r.h.s. of (5) can be evaluated numerically in a rather easy manner.

As a last example we consider the Bessel density ${ }^{1}$

$$
f_{X}(x)=\left(\frac{2}{\beta}\right)^{\nu-1} \theta^{\nu} e^{-\frac{\beta^{2}}{4 \theta}} e^{-\theta x} I_{\nu-1}(\beta \sqrt{x}) x^{\frac{\nu-1}{2}}
$$

1 The Bessel density is obtained in the following manner: consider a random variable $X$ distributed according to a gamma distribution with scale parameter $\alpha$ and mean $(\rho+k+1) / \alpha$ and take $k$ as an integer valued random variable with a Poisson distribution with parameter $\lambda$. The compound distribution of $X$ obtained by summing over $k$ has the

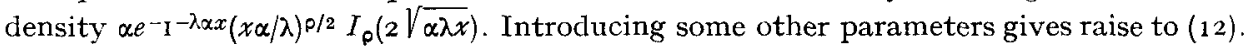


where $I_{\rho}(x)=\sum_{k=0}^{\infty} \frac{1}{k !} \frac{1}{\Gamma(\rho+k+1)}\left(\frac{x}{2}\right)^{\mathrm{i} k+\rho}$ denotes a modified Bessel function with $E(X)=\frac{1}{\theta}\left(\nu+\frac{\beta^{2}}{4^{\theta}}\right)$

$$
E\left(X^{2}\right)=\frac{1}{\theta^{2}}\left[\nu(\nu+1)+2 \frac{\beta^{2}}{4^{\theta}}(\nu+1)+\frac{\beta^{2}}{16 \theta^{2}}\right] .
$$

Making use of some of the results appearing in Gradshteyn and RyzhiK (1965) one gets:

$$
\int_{0}^{\infty} \cos (u x) f_{X}(x) d x=\frac{1}{\left(1+u^{2} / \theta^{2}\right)^{v / 2}} e^{-\frac{\beta^{2}}{4 \theta} \frac{u^{2}}{\theta^{2}+u^{2}}} \cos \left(v \operatorname{Arctg}\left(\frac{u}{\theta}\right)+\frac{\beta^{2}}{4} \frac{u}{\theta^{2}+u^{2}}\right)
$$

as well as

$$
\int_{0}^{\infty} \sin (u x) f_{X}(x) d x=\frac{1}{\left(1+u^{2} / \theta^{2}\right)^{v / 2}} e^{-\frac{\beta^{2}}{4 \theta} \frac{u^{2}}{\theta^{2}+u^{2}}} \sin \left(v \operatorname{Arctg}\left(\frac{u}{\theta}\right)+\frac{\beta^{2}}{4} \frac{u}{\theta^{2}+u^{2}}\right) .
$$

14)

And once more inserting these results into the r.h.s. of (5) gives raise to an expression for $\varphi_{F}(u)$ which is extremely elegant to compute numerically.

\section{THE NUMERICAL PROCEDURE}

Recalling (4) the numerical inversion can be reduced to one over a finite interval

$$
\int_{a}^{b} \cos (u x) \frac{1-\varphi_{F}(u)}{u^{2}} d u \quad a>0
$$

in case the remaining contributions resulting from the integration outside $[a, b]$ can be estimated with a satisfactory accuracy. But

$$
\left|\int_{b}^{\infty} \cos (u x) \frac{1-\varphi_{F}(u)}{u^{2}} d u\right| \leqslant \frac{2}{b},
$$

consequently it is sufficient to choose $b$ sufficiently large. On the other hand:

$$
\left|\int_{0}^{a} \cos (u x) \frac{1-\varphi_{F}(u)-\frac{1}{2} \lambda t\left[E\left(X^{2}\right)+\lambda t E(X)^{2}\right] u^{2}}{u^{2}} d u\right| \leqslant \frac{1}{7^{2}} a(a t)^{2} .
$$


Hence it is sufficient to choose a sufficiently small in order that

$$
\frac{1}{2} \lambda t\left[E\left(X^{2}\right)+\lambda t E(X)^{2}\right] \frac{\sin (a x)}{x}
$$

represents with the desired accuracy

$$
\int_{0}^{a} \cos (u x) \frac{1-\varphi_{F}(u)}{u^{2}} d u .
$$

The numerical procedure is based on an algorithm of Piessens and BRANders (1975) who gave an adaptive quadrative method for the automatic computation of integrals with strongly oscillating integrands. The integration of this method is based on a truncated Chebyshev series approximation. In fact the algorithm gives an adaptive quadrative method for the automatic computation of one or both of the integrals:

$$
\int_{a}^{b} \sin (w x) f(x) d x \text { and } \int_{a}^{b} \cos (w x) f(x) d x
$$

to within a user-specified absolute tolerance. This excellent algorithm has as main components

i) a procedure for evaluating

$$
S_{I_{j}}(w)=\int_{I_{j}} \sin (w x) f(x) d x
$$

where $I_{j}$ is a subinterval of $[a, b]$;

ii) a method for calculating $e_{I_{j}}(w)$, an estimate for the error

$$
\left|S_{I_{j}}(w)-\int_{I_{j}} \sin (w x) f(x) d x\right| .
$$

The interval $[a, b]$ is divided into $n$ steps where $n$ is also specified by the user. The algorithm also gives an estimate of the total error and has also a feature for detecting round-off errors. Consequently there are two possibilities for the algorithm to give wrong results, namely in case the number of steps specified by the user is not large enough, secondly in case round-off errors occur on a too large scale.

However the estimate of the error gives the possibility to decide whether the desired accuracy is reached. In our examples we have indicated with an asterisk the results that are not accurate enough, we also have written down the estimate of the error in order that the reader will be able to check that even a negative result can be obtained for the stop-loss premium in case the error estimate is larger than the absolute value of the final result. 


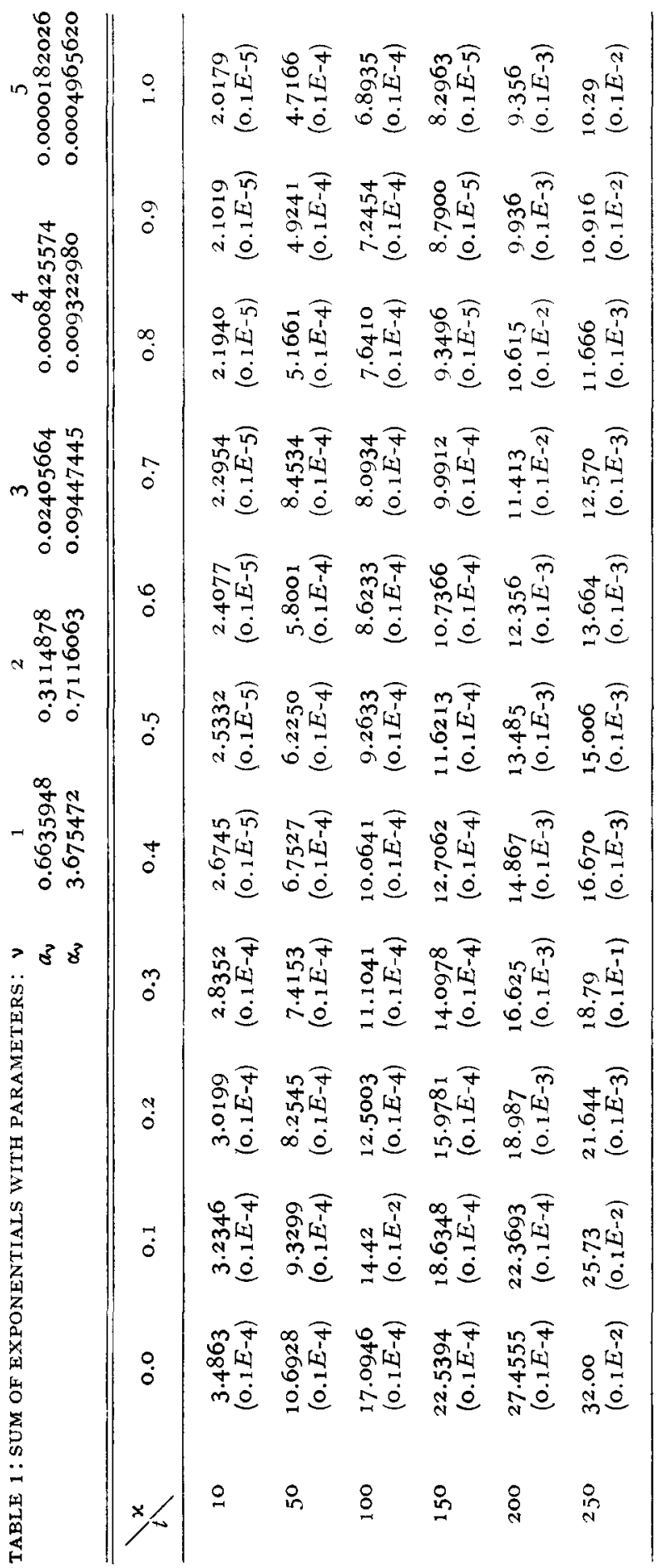




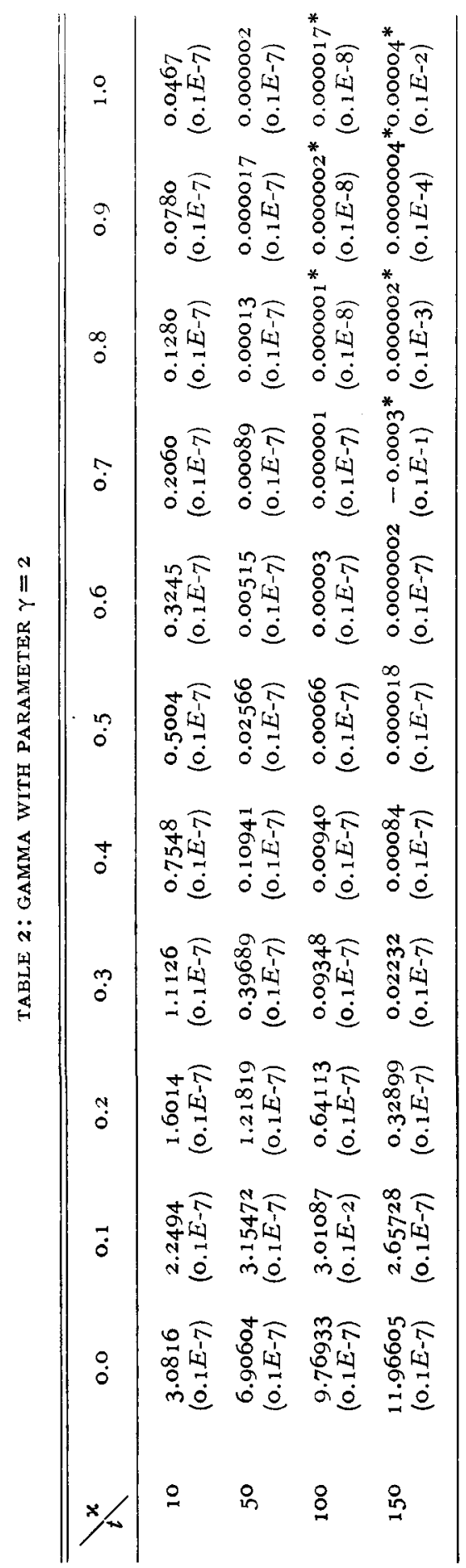

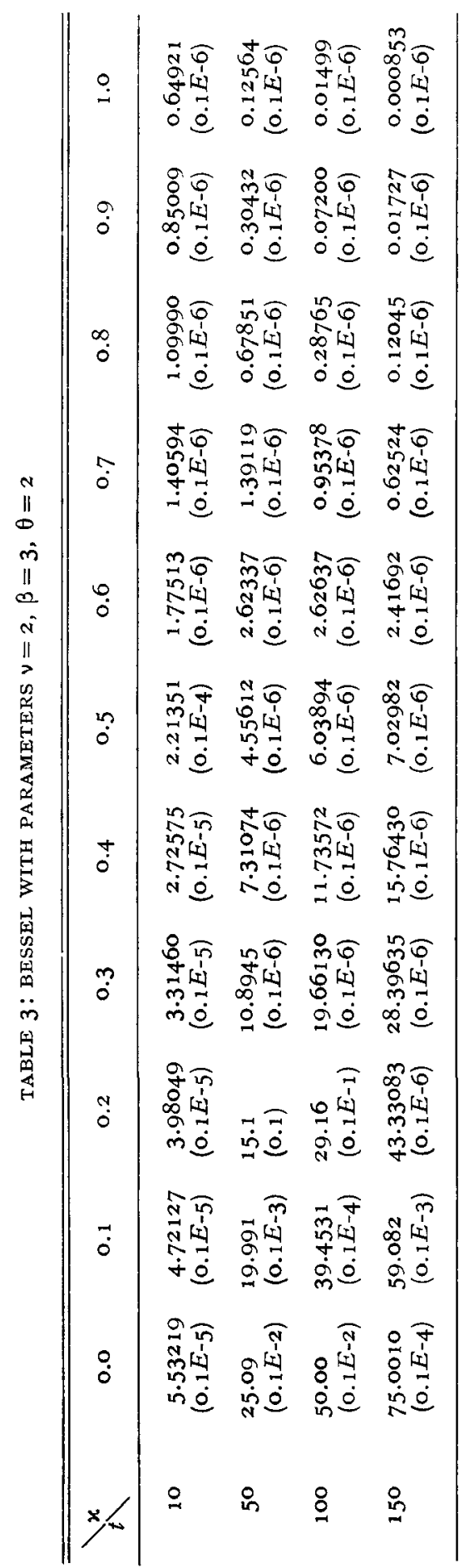




\section{NUMERICAL RESULTS}

We will give here the numerical results obtained for the three particular cases considered in section 2 .

Let the stop loss point be given by $(1+x) t E(X)$, then the following results are obtained for different values of the time parameter and for different values of $x$. An estimate of the error is given in parentheses.

REFERENCE

Bohman, H. and F. Esscher (1963). Studies in Risk Theory, Skandinavisk Aktuarietidskrift, 46, 173-225.

Gradshteyn, I. S. and I. M. Ryzhik (1965). Table of Integrals, Series and Products. New York: Academic Press.

Halmstad, D. (1976). Exact Numerical Procedures in Discrete Risk Theory, Transactions of the 2oth International Congress of Actuaries, Tokyo, 557-562.

Pressens, R. and M. Branders (1975). Algorithm oo2, Computation of Oscillating Integrals, Journal of Computational and Applied Mathematics 1, 153-164.

SEAJ, H. (1977). Numerical Inversion of Characteristic Functions, Scandinavian Actuarial Journal 5, 48-53.

Thorin, O. and N. Wikstad (1976). Calculation and Use of Ruin Probabilities, Transactions of the 2oth International Congress of Actuaries, Tokyo, 773-781.

Institute of Actuarial Science.

Catholic University Louvain, Louvain, Belgium. 